\title{
Cardiac Function and Diastolic Dysfunction in Behcet's Disease: A Systematic Review and Meta-Analysis
}

\author{
Fawad Aslam, ${ }^{1}$ Salman J. Bandeali, ${ }^{2}$ Cynthia Crowson, ${ }^{3}$ and Mahboob Alam ${ }^{4}$ \\ ${ }^{1}$ Division of Rheumatology, Mayo Clinic Health System, Eau Claire, WI 54703, USA \\ ${ }^{2}$ Department of Cardiology, Texas Heart Institute at CHI St. Luke's Medical Center, Houston, TX 77030, USA \\ ${ }^{3}$ Division of Biomedical Statistics and Informatics, Department of Health Sciences Research and Division of Rheumatology, \\ Department of Internal Medicine, Mayo Clinic, Rochester, MN 55906, USA \\ ${ }^{4}$ Section of Cardiology, Department of Medicine, Baylor College of Medicine, Houston, TX 77030, USA
}

Correspondence should be addressed to Mahboob Alam; mahboob.alam@bcm.edu

Received 20 February 2016; Accepted 13 April 2016

Academic Editor: Malcolm Smith

Copyright (C) 2016 Fawad Aslam et al. This is an open access article distributed under the Creative Commons Attribution License, which permits unrestricted use, distribution, and reproduction in any medium, provided the original work is properly cited.

Background. Cardiovascular involvement in Behcet's disease (BD) is reported and has variable manifestations. It is not clear if diastolic dysfunction (DD) is increased in BD. Our objective was to evaluate the existing literature to determine if cardiac dysfunction, particularly DD, was more prevalent in these patients. Methods. A systematic review and meta-analysis of the available studies analyzing the echocardiographic findings in BD was conducted using a random-effects model. Mean differences were used to calculate the effect sizes of the echocardiographic parameters of interest. Results. A total of 22 studies with 1624 subjects were included in the analysis. Patients with BD had statistically significantly larger mean left atrial dimension $(0.08, p=0.0008)$, greater aortic diameter $(0.16, p=0.02)$, significantly reduced ejection fraction $(-1.08, p<0.0001)$, significantly prolonged mitral deceleration time $(14.20, p<0.0001)$, lower $E / A$ ratio $(-0.24, p=0.05)$, and increased isovolumetric relaxation time (7.29, $p<0.00001)$. Conclusion. DD is increased in patients with BD by the presence of several echocardiographic parameters favoring $\mathrm{DD}$ as compared to controls. The meta-analysis also identified that LA dimension is increased in BD patients. EF has also been found to be lower in $\mathrm{BD}$ patients. Aortic diameter was also increased in $\mathrm{BD}$ patients as compared to controls.

\section{Introduction}

Behcet's disease $(\mathrm{BD})$ is a chronic inflammatory disease. $\mathrm{BD}$ is seen throughout the world and its estimated incidence ranges from 1 per 100,000 to 70 per 100,000 depending on the geographic region $[1,2]$. It can virtually involve any organ system. Cardiac involvement is variable and thought to occur in the range of 7-46\% [3] of patients and may cause myocardial infarction, pericarditis, valve problems, aneurysms, or congestive heart failure (CHF).

The development of $\mathrm{CHF}$ in $\mathrm{BD}$ in itself is rare and secondary to vasculitis which leads to impaired pump function [4]. The fact that patients with BD are relatively young without traditional risk factors and yet can develop these potentially fatal complications merits closer scrutiny and the need to be able to identify the precursor forms of these manifestations.

CHF is a well-known clinical entity which is diagnosed by a combination of clinical criteria and measurements on transthoracic echocardiogram (TTE). Left ventricular diastolic dysfunction (DD) is an important element of CHF as DD may present with its own signs and symptoms or may present as a precursor to overt CHF [5]. The presence of DD in the presence of normal ejection fraction (EF) and presence of symptoms of heart failure is known as diastolic heart failure or as heart failure with preserved ejection fraction [6]. The isolated presence of diastolic mechanical abnormalities on TTE is termed DD and is characterized as one of the stages of heart failure and a target of intervention to prevent progressive heart failure [7]. 
DD is not a rare disease entity. In the general population, a population based study of more than two thousand research participants reported an overall DD prevalence of $28.1 \%$ [8]. Another research paper involving over 36,000 subjects had a DD prevalence of $65.1 \%$ with the most common form being that of mild DD (60.0\%) [9]. In the general population, DD has been identified as an independent predictor of new onset $\mathrm{CHF}[10]$. It is also a standalone determinant of mortality $[8,9,11]$.

DD is a collection of mechanical problems in the contraction and relaxation of the heart. DD has been found to occur in association with diabetes mellitus (DM), increasing age, coronary artery disease (CAD), hypertension (HTN), and overweight/obesity [12]. Increased left atrial (LA) dimension also indicates DD provided there are no concomitant rhythm disorders [11, 13]. Diastolic dysfunction is an echocardiographic diagnosis and not a clinical one.

There is recent literature to support increased cardiac problems in other common rheumatic diseases like rheumatoid arthritis. One recent paper has shown increased DD in rheumatoid arthritis [14]. The data regarding the presence of $\mathrm{DD}$ in $\mathrm{BD}$ is controversial. Most of these studies were of case-control design and small in size. Our objective was to gather and analyze the available data through a metaanalysis to compare the prevalences of CHF and DD, based on TTE parameters, in patients with BD as compared to healthy controls to try to answer the question of this association more definitively.

\section{Methods}

2.1. Literature Search Strategy. The review was conducted according to the recommendations of the meta-analysis of observational studies in epidemiology (MOOSE) group [37]. A time restricted (year 1990 and onwards) and language restricted (English) Medline search with the medical subject heading (MESH) terms "(heart failure OR ventricular dysfunction OR atrial OR echocardiography) AND (behcet OR behcet's disease)" was conducted on 31 December 2013. We also perused the bibliography of the identified studies as well as general review articles on BD to identify any appropriate studies that may have been not selected in the search. Unpublished studies were not included. Bibliographically identified studies were only included if they were also retrievable from the Medline database.

A non-English language search was also performed using the same search strategy but utilizing the language filter properties of Medline. The goal of the non-English language study search was to only review the abstracts and compare the conclusions regarding echocardiographic parameters from the abstracts.

2.2. Study Selection. The studies were independently selected by two researchers using our predetermined search strategy. Any differences were resolved by mutual consultation. Studies performed with the intent of evaluating cardiac function between BD patients and controls were included. Studies were only included if the identified patients with $\mathrm{BD}$ meet the diagnostic criteria of the international study group for BD [38]. Only case-control studies were included as they provided an odd-ratio estimate of study differences between BD patients and the general population. We included only those studies that had adjusted for or excluded patients with preexisting cardiac conditions. This was to ensure minimal confounding. Studies including patients with overlapping rheumatic disorders were excluded from the study as other rheumatic conditions are known to affect the heart and may confound the results.

Non-English language Medline search revealed 39 studies. Of all these studies only one title merited further scrutiny; however no abstract was available for review.

2.3. Study Quality. The Newcastle-Ottawa scale (NOS) for case-control studies was used to assess the quality of the included studies [39]. A higher score is indicative of better methodological quality.

2.4. Data Collection. Data was collected pertaining to the sociodemographic, BD related, and laboratory variables. TTE variables included EF in percent (\%), isovolumetric relaxation time (IVRT) in millisecond ( $\mathrm{msec}$ ), and mitral valve deceleration time (DT) in msec, transmitral early filling peak velocity $(E)$ in meters per second $(\mathrm{m} / \mathrm{sec})$, transmitral late filling peak velocity $(A)$ in $\mathrm{m} / \mathrm{sec}$, and the $E / A$ ratio. Other variables, where available, included left atrial dimension (LAD) in centimeter $(\mathrm{cm})$, left ventricular end diastolic dimension (LVDD) in $\mathrm{cm}$, left ventricular end systolic dimension (LVSD) in $\mathrm{cm}$, aortic diameter in $\mathrm{cm}$, and aortic distensibility in $\mathrm{cm}^{2} \times$ dyn. Summary estimates were used as individual level patient data was not available.

While the comparison of EF and aortic size was fairly straightforward, that is not the case for DD. A variety of combinations or single values of the aforementioned variables are used to classify patients with DD [40]. The more supportive the variables, the higher the likelihood of having DD. We kept a low threshold and even if one parameter was favoring DD, the subjects were classified as so. It is also to be noted that $\mathrm{DD}$ is graded in terms of the degree of impairment into three categories. Such information was not available to us regarding grades of DD.

2.5. Statistical Methodology. Since individual patient information was not available for all patients, we report weighted means and standard deviations. Percentages and means \pm standard deviations (SD) were calculated to describe the distributions of categorical and continuous variables, respectively. Student's $t$-test for independent samples was used for continuous variables and chi-square test for proportions. The baseline data were analyzed using SAS version 9.3 (SAS Institute, Cary, NC).

Review Manager, version 5.2 (Cochrane Collaboration), was used for the statistical computations of this study. Mean differences (MD) and their 95\% confidence intervals (95\% CIs) were calculated to determine the effect size for each parameter measured via TTE using the random-effects model. Heterogeneity measurements were calculated and 


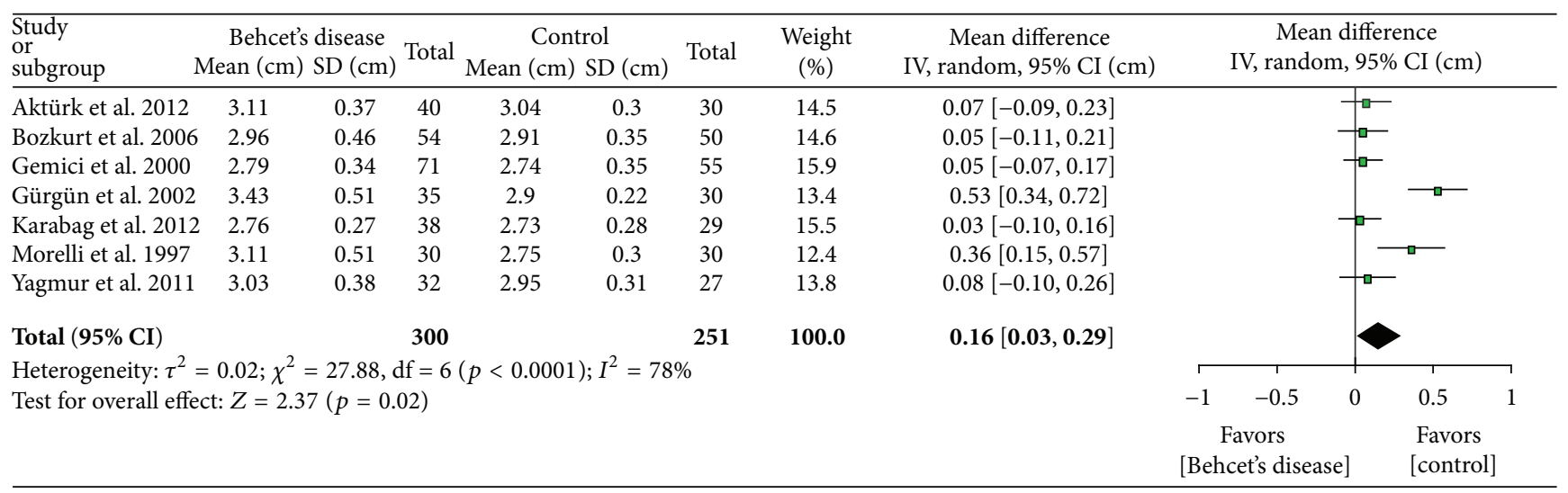

FIGURE 1: Aortic size comparison between patients with Behcet's disease and controls.

reported by including Cochran's Q-statistic, the $I^{2}$ index, and the tau-squared test. Random-effects model was used to calculate the MD and 95\% CI. This was done due to the range of heterogeneity in the data.

\section{Results}

A total of 185 citations were identified based on the aforementioned search strategy. Of these, 22 were selected after abstract review (Table 1). A total of 1624 subjects were identified of which $913(56 \%)$ had BD. The mean BD duration was 8.2 years. Pulse wave Doppler (PWD) technique was the most commonly used technique to assess TTE parameters but the newer studies used tissue Doppler imaging (TDI) technique. Mean disease duration was 8.2 years. Mean age of the cases was 37.7 years and that of controls was 37.3 years. Of the cases, $60 \%$ were males and $77 \%$ of the controls were males. No difference on the basis of smoking, DM, HTN and hyperlipidemia, lipid values, and systolic blood pressure (SBP) was noted. Body mass index (BMI) was statistically significantly higher in the control population (24.8 versus $24.1 \mathrm{~kg} / \mathrm{m}^{2}, p=0.037$ ). High density lipoprotein (HDL) was significantly higher in the control population (43.5 versus $41.4 \mathrm{mg} / \mathrm{dL}, p=0.05$ ) but was likely clinically insignificant based on the small difference. Diastolic blood pressure (DBP) was significantly higher in the cases $(76.8$ versus $74.8 \mathrm{mmHg}$, $p<0.001)$. C-reactive-protein (CRP) was significantly higher in the cases (18.1 versus $8.8 \mathrm{mg} / \mathrm{dL}, p<0.001$ ) as expected.

All studies had participants that were matched on the basis of age and sex and as a general exclusion criterion; subjects with CAD, DM, HTN, and other heart diseases were excluded. Racial data was not available for the studies. The average quality score was 6.4 of a maximum possible of 9 on the NOS for the included studies. Of the 22 studies, 17 showed some difference in TTE parameters between the two groups while 5 showed similar indices. Table 1 gives the details of the studies comprehensively.

Most studies (16 out of 22) reported on medications used in DD patients. Patients on medications known to affect the cardiac functional parameters were usually excluded. As we performed aggregate level data meta-analysis and no individual patient data was reported, we are unable to comment on the effects of medication usage on DD.

Table 2 shows the main meta-analysis outcomes. Comparison of the $E / A$ ratios (figure not shown) shows lower values $(-0.24, p=0.05)$ in $\mathrm{BD}$ patients which is an indicator of DD. As expected, aortic diameter (Figure 1) was found to be greater $(0.16 \mathrm{~cm}, p=0.02)$ in patients with $\mathrm{BD}$ than in controls. Larger diameter predisposes to aneurysms. Transmitral $E$ velocity (figure not shown) was found to be lower in $\mathrm{BD}$ patients $(-0.03 \mathrm{~cm}, p=0.07)$ suggestive of DD. IVRT (Figure 2) was increased in BD patients $(7.29 \mathrm{~m} / \mathrm{s}$, $p<0.00001)$ suggesting impaired cardiac relaxation and pointing towards DD. Mitral DT was found to be statistically significantly prolonged $(14.20 \mathrm{msec}, p<0.0001)$ in $\mathrm{BD}$ patients (figure not shown). LAD was found to be statistically significantly larger $(0.08 \mathrm{~cm}, p=0.0008)$ in $\mathrm{BD}$ patients (Figure 3) suggesting DD. This is also a risk factor for atrial fibrillation [41]. EF of the left ventricle was found to be statistically significantly reduced $(-1.08 \%, p<0.0001)$ in $\mathrm{BD}$ patients (Figure 4) suggesting impaired cardiac function and pumping ability in BD patients as compared to controls.

In terms of other echocardiographic variables, no statistically significant differences were noted between left ventricular mass index, LVSD, LVDD, posterior wall thickness, and left ventricular myocardial perfusion index. With regard to the aortic distensibility, which measures the stiffness of the aortic wall, while the two individual studies [23,27] reporting this parameter show statistically significant reduced aortic distensibility (figure not shown), the composite outcome was not significant due to the fact that the first study has a much larger effect size and a higher variance than the second study. This heterogeneity leads to a wide confidence interval for the combined results and thus a nonsignificant value.

\section{Discussion}

This systematic review and meta-analysis looking at data from 1624 subjects has clearly shown the presence of DD indices on TTE is greater in patients with $\mathrm{BD}$ than with controls. These indices include $E$ wave velocity, $E / A$ ratio, IVRT, and mitral DT. Prolonged mitral DT and IVRT were the most 


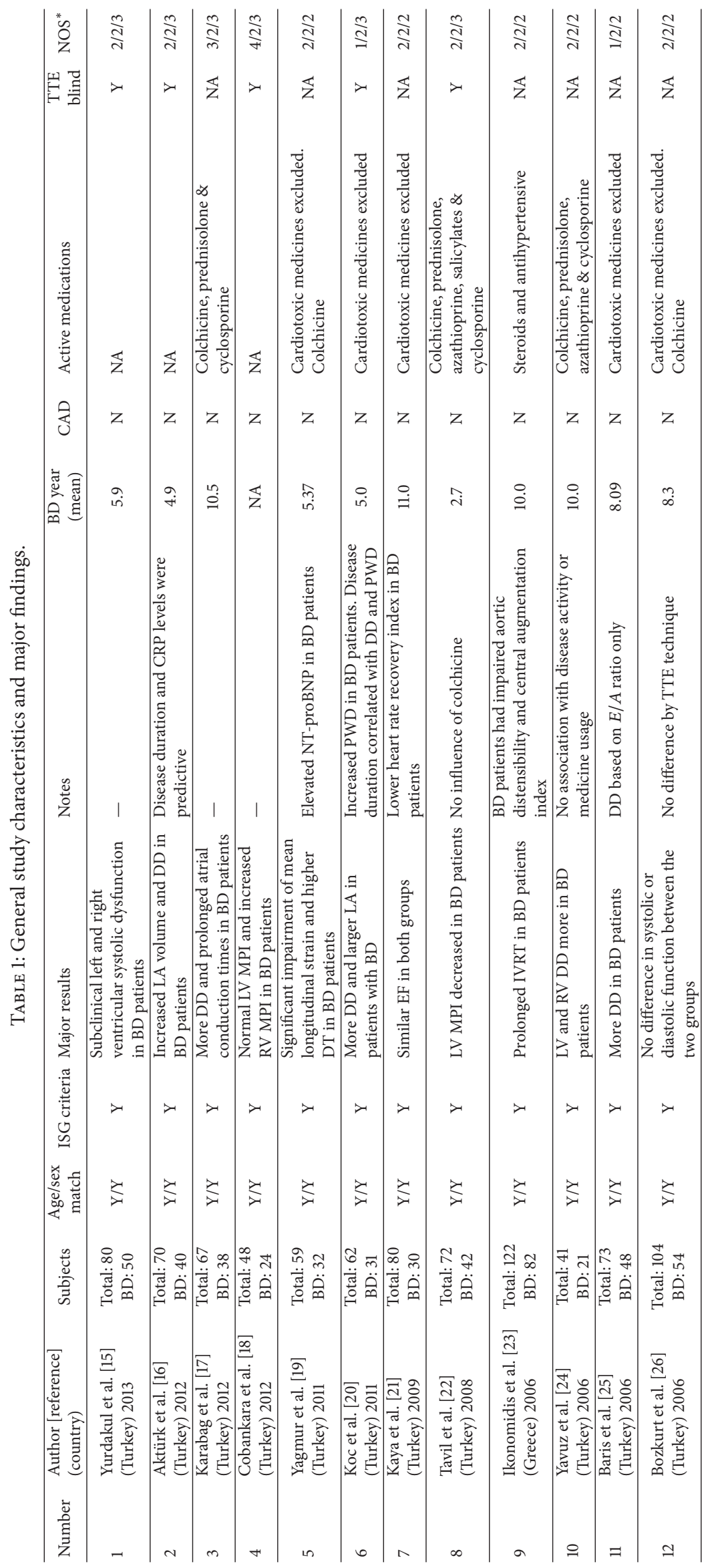




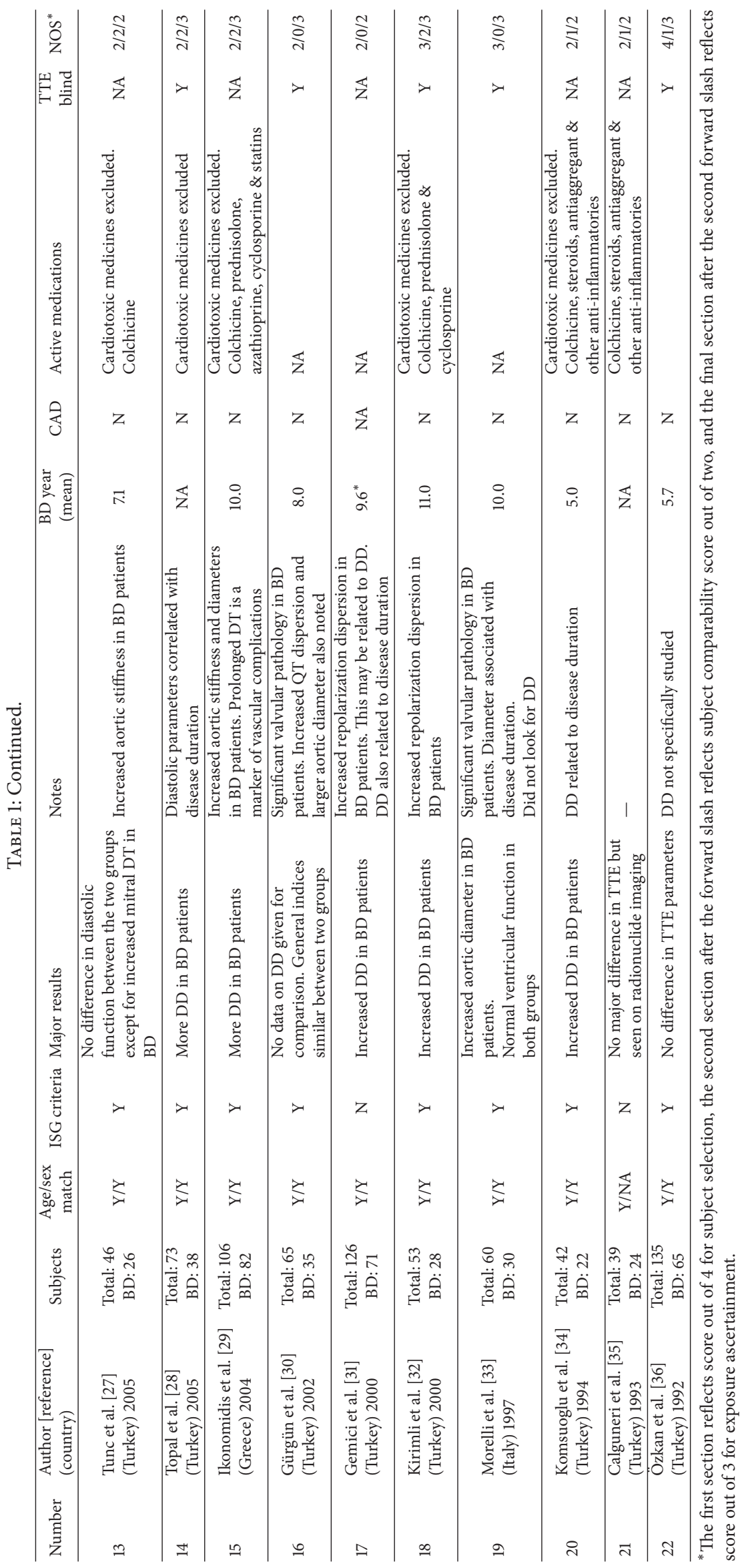


TABLE 2: Meta-analysis outcomes (random-effects model).

\begin{tabular}{|c|c|c|c|c|c|}
\hline Variables & Mean difference $(95 \% \mathrm{CI})$ & $p$ & $Q^{* *}$ & $I^{2}, \%^{\ddagger}$ & $\tau^{2^{\S}}$ \\
\hline Left atrial dimension, $\mathrm{cm}(n=958)$ & $0.08(0.03,0.12)$ & 0.0008 & 11.13 & 0 & 0.00 \\
\hline Left ventricular mass index, $\mathrm{gm} / \mathrm{m}^{2}(n=300)$ & $3.53(-1.08,8.14)$ & 0.13 & 0.11 & 0 & 0.00 \\
\hline Isovolumetric relaxation time, $\mathrm{msec}(n=1023)$ & $7.29(4.86,9.72)$ & $<0.0001$ & 18.48 & 35 & 6.64 \\
\hline Transmitral $A$ wave velocity, meters/second $(n=705)$ & $0.03(-0.01,0.08)$ & 0.18 & 37.21 & 79 & 0.00 \\
\hline Transmitral $E$ wave velocity, meters/second $(n=705)$ & $-0.03(-0.06,0.00)$ & 0.07 & 15.04 & 47 & 0.00 \\
\hline$E / A$ ratio $(n=304)$ & $-0.24(-0.48,0.00)$ & 0.05 & 36.50 & 89 & 0.06 \\
\hline Mitral deceleration time, $\operatorname{msec}(n=1023)$ & $14.20(7.67,20.73)$ & $<0.0001$ & 32.96 & 34 & 82.96 \\
\hline Left ventricular ejection fraction, $\%(n=1280)$ & $-1.08(-1.58,-0.58)$ & $<0.0001$ & 15.14 & 0 & 0.00 \\
\hline Posterior wall, $\mathrm{cm}(n=682)$ & $0.00(-0.03,0.03)$ & 0.81 & 25.01 & 64 & 0.00 \\
\hline Left ventricular end systolic dimension, $\mathrm{cm}(n=742)$ & $0.03(-0.04,0.10)$ & 0.37 & 18.03 & 45 & 0.01 \\
\hline Left ventricular end diastolic dimension, $\mathrm{cm}(n=874)$ & $-0.02(-0.08,0.04)$ & 0.46 & 16.31 & 26 & 0.00 \\
\hline BSA, $\mathrm{m}^{2}(n=80)$ & & 0.12 & & & \\
\hline LV MPI $(n=200)$ & $0.04(-0.07,0.16)$ & 0.48 & 47.96 & 96 & 0.01 \\
\hline Aorta, cm $(n=551)$ & $0.16(0.03,0.29)$ & 0.02 & 27.88 & 78 & 0.02 \\
\hline Aortic distensibility $(n=168)$ & $-1.23(-3.06,0.60)$ & 0.19 & 27.69 & 96 & 1.69 \\
\hline Systolic blood pressure, $\mathrm{mmHg}(n=994)$ & $1.69(0.16,3.22)$ & 0.03 & 14.59 & 11 & 0.92 \\
\hline Diastolic blood pressure, $\mathrm{mmHg}(n=941)$ & $1.34(0.29,2.39)$ & 0.01 & 12.69 & 5 & 0.20 \\
\hline Heart rate $(n=927)$ & $0.68(-0.76,2.13)$ & 0.35 & 29.14 & 59 & 2.85 \\
\hline Males, \% $(n=1199)$ & $0.96(0.87,1.06)^{*}$ & 0.39 & 4.16 & 0 & 0.00 \\
\hline Age, mean years $(n=1238)$ & $-0.40(-1.32,0.53)$ & 0.40 & 17.98 & 5 & 0.22 \\
\hline BMI, $\mathrm{kg} / \mathrm{m}^{2}(n=831)$ & $-0.27(-0.82,0.27)$ & 0.32 & 16.32 & 39 & 0.29 \\
\hline Diabetes mellitus $(n=295)$ & $1.31(0.42,4.05)^{*}$ & 0.64 & 0.17 & 0 & 0.00 \\
\hline Hypertension $(n=373)$ & $0.92(0.52,1.65)^{*}$ & 0.79 & 0.30 & 0 & 0.00 \\
\hline Hyperlipidemia $(n=154)$ & $1.15(0.50,2.64)^{*}$ & 0.74 & 0.62 & 0 & 0.00 \\
\hline Smoking $(n=728)$ & $0.97(0.83,1.15)^{*}$ & 0.76 & 5.04 & 0 & 0.00 \\
\hline Low density lipoprotein, mg/dL $(n=465)$ & $-0.72(-7.48,6.05)$ & 0.84 & 22.89 & 74 & 54.06 \\
\hline $\mathrm{HDL}, \mathrm{mg} / \mathrm{dL}(n=433)$ & $-0.91(-3.38,1.56)$ & 0.47 & 13.30 & 55 & 5.44 \\
\hline Triglycerides, mg/dL $(n=550)$ & $-1.35(-10.86,8.16)$ & 0.78 & 11.79 & 49 & 72.83 \\
\hline Total cholesterol, mg/dL $(n=637)$ & $-0.65(-7.16,5.85)$ & 0.84 & 13.91 & 42 & 36.40 \\
\hline HS-CRP, mg/dL $(n=268)$ & $7.79(1.89,13.68)$ & 0.01 & 10.47 & 71 & 24.27 \\
\hline
\end{tabular}

${ }^{*}$ Risk ratio.

${ }^{* *}$ Cochran's Q-statistic for heterogeneity.

${ }^{\ddagger} I^{2}$ index for degree of heterogeneity.

${ }^{\S}$ Tau-squared measure of heterogeneity.

strongly linked DD parameters on TTE in DD patients with BD. Three more important statistically significant findings were that of increased LA size, depressed EF, and increased aortic dimensions in patients with $\mathrm{BD}$ as compared to the controls.

The finding of depressed EF reveals the likely presence of a pattern of pathology affecting both systolic and diastolic parameters of the heart. N-terminal brain natriuretic peptide has been found to be elevated in patients with $\mathrm{BD}$ signifying the heart strain and elevated risk of cardiac pump problems $[42,43]$. This data provides further corroboration of the TTE findings in our study. It must be noted that although the EF was lower in cases as compared to controls, it was not necessarily abnormal.

Increased aortic diameter was seen in this meta-analysis. There are reports of aortitis [44] and inflammation involving the root of the aorta which can lead to subsequent aneurysm formation. Aneurysms can be a major cause of death if they rupture. This finding stresses the importance of following up these patients for further dilation of the aortic root and to take prophylactic measures, if indicated.

One reason for seeing DD in otherwise asymptomatic patients could be a result of the microvascular inflammation that has been seen in cardiac exam and perfusion imaging studies in BD patients $[45,46]$. These may affect the hearts mechanical properties through local inflammation and tissue remodeling causing altered TTE indices. Coronary artery vasculitis might also be a contributing factor to this impaired performance through inducing micro level ischemia.

Arterial stiffness is a reliable predictor of cardiovascular mortality and morbidity. It can be assessed by pulse wave velocity (PVW). Central systolic and diastolic pressure can be determined via pulse wave analysis (PWA). An important aspect of wave analysis is wave reflection. Wave reflection depicts the interaction of the outgoing impulse of the cardiac pressure and the reflection from the vascular system. PVW 


\begin{tabular}{|c|c|c|c|c|c|c|c|c|c|c|}
\hline $\begin{array}{l}\text { Study } \\
\text { or } \\
\text { subgroup }\end{array}$ & \multicolumn{2}{|c|}{ Behcet's disease } & Total & \multicolumn{2}{|c|}{ Control } & Total & $\begin{array}{l}\text { Weight } \\
(\%)\end{array}$ & $\begin{array}{c}\text { Mean difference } \\
\mathrm{IV} \text {, random, } 95 \% \mathrm{CI}(\mathrm{m} / \mathrm{s})\end{array}$ & \multicolumn{2}{|c|}{$\begin{array}{l}\text { Mean difference } \\
\mathrm{IV} \text {, random, } 95 \% \mathrm{CI}(\mathrm{m} / \mathrm{s})\end{array}$} \\
\hline Bozkurt et al. 2006 & 80.8 & 16.1 & 54 & 83 & 13.6 & 50 & 10.1 & $-2.20[-7.91,3.51]$ & $\longrightarrow$ & \\
\hline Yagmur et al. 2011 & 87.96 & 11.2 & 32 & 83.33 & 9.5 & 27 & 11.1 & $4.63[-0.65,9.91]$ & & \\
\hline Tavil et al. 2008 & 91 & 19 & 42 & 85 & 15 & 30 & 6.8 & $6.00[-1.86,13.86]$ & & \\
\hline Baris et al. 2006 & 96.4 & 13.8 & 48 & 90.2 & 12.5 & 26 & 9.2 & $6.20[0.01,12.39]$ & & \\
\hline Tunc et al. 2005 & 99.6 & 20.6 & 26 & 93.2 & 17.8 & 20 & 4.0 & $6.40[-4.72,17.52]$ & & \\
\hline Koc et al. 2011 & 88 & 21 & 31 & 81 & 18 & 31 & 4.9 & $7.00[-2.74,16.74]$ & & \\
\hline Topal et al. 2005 & 95 & 17 & 38 & 88 & 15 & 35 & 7.4 & $7.00[-0.34,14.34]$ & & \\
\hline Karabag et al. 2012 & 90 & 23 & 38 & 82 & 18 & 29 & 4.8 & $8.00[-1.82,17.82]$ & & \\
\hline Komsuoglu et al. 199 & 9490.6 & 26 & 22 & 81.4 & 14.2 & 20 & 3.2 & $9.20[-3.32,21.72]$ & & \\
\hline Ikonomidis et al. 200 & $04 \quad 87$ & 15 & 82 & 77 & 19 & 24 & 6.3 & $10.00[1.73,18.27]$ & & \\
\hline Aktürk et al. 2012 & 89.87 & 11.48 & 40 & 79.63 & 7.69 & 30 & 12.9 & $10.24[5.74,14.74]$ & & \\
\hline Gemici et al. 2000 & 77.81 & 20.52 & 71 & 66.72 & 12.44 & 55 & 10.0 & $11.09[5.29,16.89]$ & & \\
\hline Ikonomidis et al. 200 & 0687 & 15 & 82 & 74.4 & 17 & 40 & 9.2 & $12.60[6.41,18.79]$ & & \\
\hline Total $(95 \%$ CI $)$ & & & 606 & & & 417 & 100.0 & $7.29[4.86,9.72]$ & & \\
\hline \multicolumn{9}{|c|}{$\begin{array}{l}\text { Heterogeneity: } \tau^{2}=6.64 ; \chi^{2}=18.48, \mathrm{df}=12(p=0.10) ; I^{2}=35 \% \\
\text { Test for overall effect: } Z=5.88(p<0.00001)\end{array}$} & $-20-10 \quad 0$ & $10 \quad 20$ \\
\hline & & & & & & & & & $\begin{array}{l}\text { Favors } \\
\text { [Behcet's disease] }\end{array}$ & $\begin{array}{l}\text { Favors } \\
{[\text { control }]}\end{array}$ \\
\hline
\end{tabular}

FIGURE 2: Isovolumetric relaxation time comparison between patients with Behcet's disease and controls.

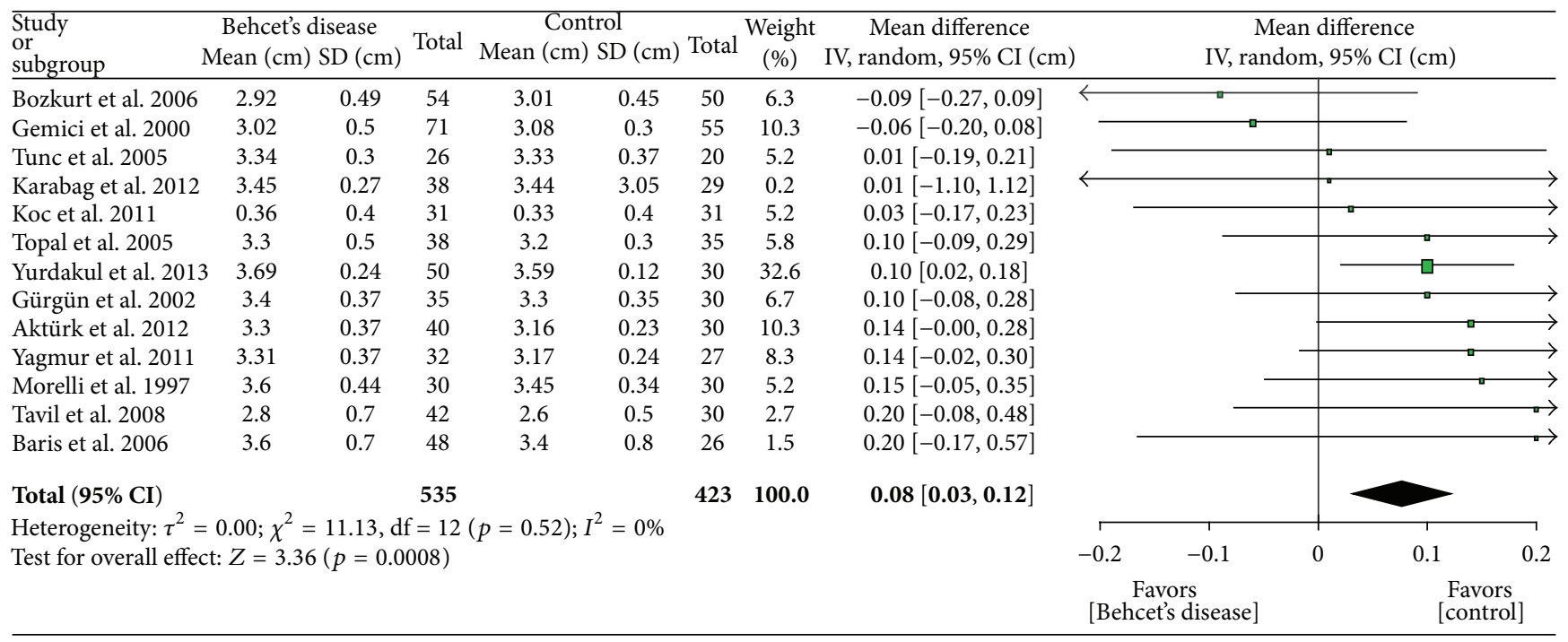

FIGURE 3: Left atrial dimension comparison between patients with Behcet's disease and controls.

is a marker of arterial stiffness. It has been found to be elevated in patients with BD who have active disease [47]. Nitric oxide (NO) serves as a vasodilator. It is thought that inflammation mediated endothelial dysfunction in BD contributes to increased arterial stiffness. Inflammation mediated release of reactive oxygen species further interferes with NO release and also causes stiffness of cardiac myocytes [6].

It is well known that most of the intrinsic blood flow to the heart occurs during diastole. This is due to the compression of the surrounding musculature. Coronary flow reserve (CFR) is a way to assess the microcirculation of the heart and thereby the endothelial function. This assessment is usually via echocardiography. It is defined as maximal coronary flow divided by basal flow. It can be altered by epicardial pathology and/or microvascular dysfunction. Atherosclerosis is known to have an inflammatory component. Systemic inflammation was thought to play a role in the reduction of CFR in psoriasis patients without any known coronary disease [48]. Reduced CFR has also been found in patients with BD [49], RA [50], and scleroderma [51] thus suggesting the underlying role of inflammation.

The natural history of DD is not very well defined. Studies on the common populace have shown that the factors promoting DD progression to symptomatic stages include ischemia, rhythm problems like atrial fibrillation, hypertension, or hospitalization but remain unknown in 50\% of cases [52].

Data from one study of the general population revealed that the two-year likelihood of developing systolic CHF was 


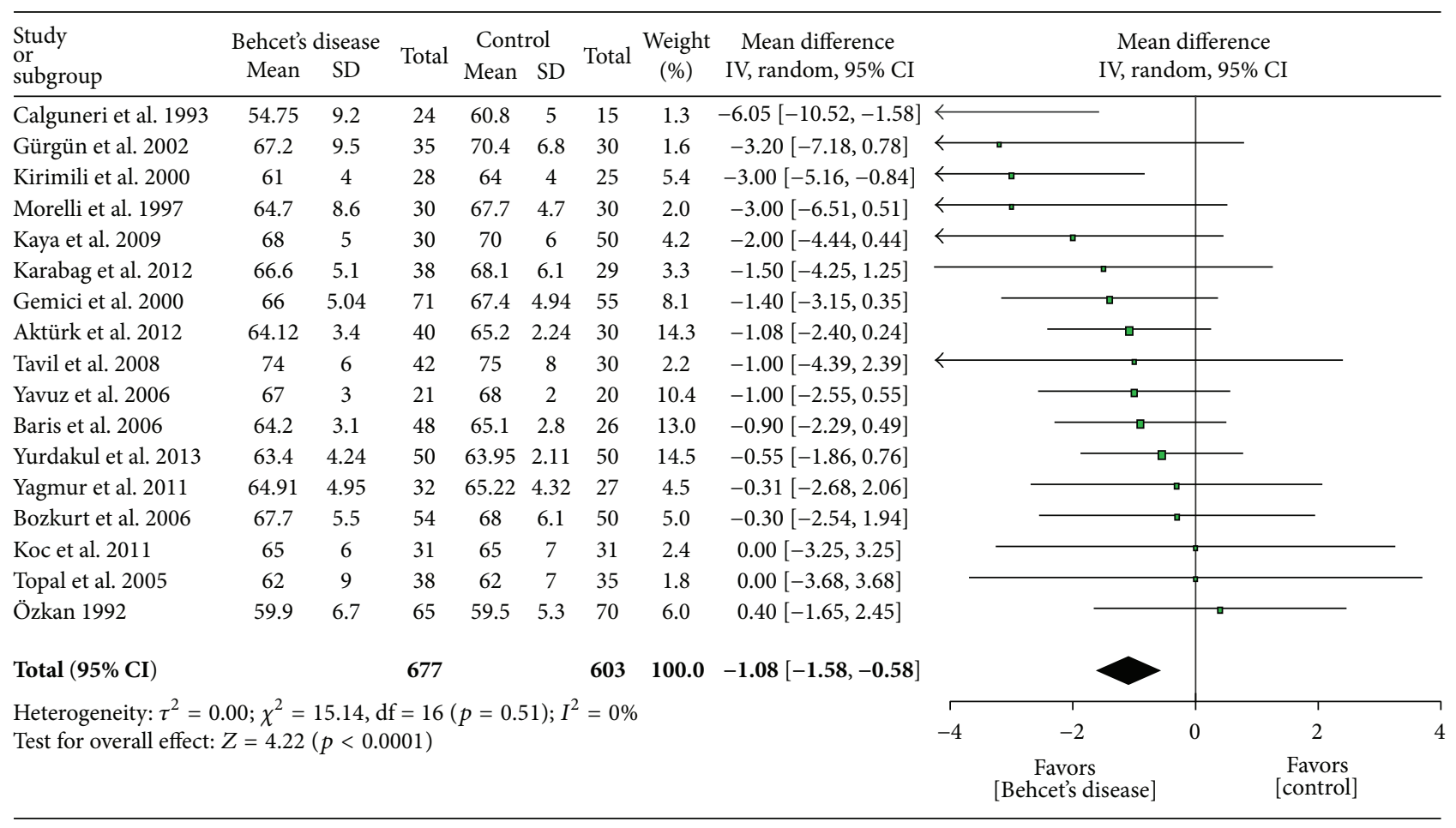

FIGURE 4: Left ventricular ejection fraction comparison between patients with Behcet's disease and controls.

$1.9 \%$ while the likelihood of developing clinical manifestations of heart failure in patients already suffering from DD was at $31.1 \%$ [52]. One general population report identified that TTE parameters of DD remained steady in about $50 \%$ of the patients, showed improvement in $21 \%$, and based on clinical symptoms deteriorated in $27 \%$ after observation for over 3.6 years; and importantly improvement to a less severe degree of DD conferred a survival benefit [11].

There were several studies which assessed the relationship of $\mathrm{BD}$ duration to the presence of DD. As is theoretically expected because of accruing damage from the inflammation over the years as well as advancing age promoting coexisting problems, most of these studies reported association of DD and/or aortic diameter with longer BD duration $[16,20,22$, $28,31,33,34]$. One study reported on the relationship between the activity state of $\mathrm{BD}$, whether disease was controlled or not, and cardiac manifestations but no significant association was found in that report [24].

The result of increased LA dimension is of great potential importance, because increased LA dimension is a risk factor for atrial fibrillation $[41,53]$ which in turn confers a high risk of suffering from a stroke. LA dimensions and mechanical problems have also been identified in other autoimmune diseases pointing to a unifying final pathway in these conditions $[14,54]$. At present, there is no population level data about the association between atrial fibrillation and $\mathrm{BD}$, but this study has certainly identified an important area for future original research from a clinical health perspective.

Another factor of importance was the concept of electromechanical delay (EMD) identified in one of the studies of this meta-analysis [17]. This concept basically implies that it takes a longer time for the electrical energy generated in the heart muscle to transform into mechanical energy which then leads to the contraction of the heart. Increased EMD is a wellestablished predictor of development of atrial fibrillation [55]. Studies have previously also shown findings of prolonged atrial signal travelling time in $\mathrm{BD}$ cases which was related to the length of their disease process [56]. The finding of increased EMD in $\mathrm{BD}$ also further corroborates the potential for increased risk of atrial arrhythmia [17]. It is believed that the vasculitis process in BD leads to fibrosis of the cardiac and atrial muscles which interferes with the passage of electrical signal in the atrial musculature leading to aberrant circuit formation [17]. Measuring EMD by TDI may be a simple and useful way to analyze the risk of atrial fibrillation in patients with BD.

4.1. Treatment Implications and Suggestions for Future Research. At present there is no targeted and specific treatment for DD. The focus is on the control of the risk factors that may lead to DD and its progression to overt CHF. Studies are underway to determine appropriate treatment strategies for these patients [5]. Aggressive blood pressure control [57] may improve TTE diastolic indices [58]. It is important for physicians managing patients with $\mathrm{BD}$ to consider their higher risk of developing DD and CHF and be more aggressive in the control of traditional risk factors if present. However, since BD is relatively a disease of the young and these patients may not have developed the traditional risk factors thus their cardiac health may not be assessed. At present, it is not clear whether these risk factors should be more actively sought in these patients or not. Also it is 
not clear as to how to follow up these patients, especially if asymptomatic, with echocardiograms, and if they do have subtle findings, how to go about addressing them. These are important areas for future research and designing of health services. Further future studies could look at the activity of the disease and relate it to development of cardiac problems. Additional studies could be designed to compare BD patients who develop cardiac complications with those who do not and try to ascertain the determinants of the differences.

4.2. Strengths of the Study. Our analysis included mostly subjects without any of the known medical conditions that are risk factors for DD. All studies were of decent quality according to the NOS scale quality and had the primary objective planned to evaluate TTE parameters in BD. It is important to note that most studies had excluded patients with known cardiotoxic medications which could have confounded the results. One strength of our data is that it has identified many TTE parameters, rather than just one, pointing towards DD in these patients and this lends strength to the concept that DD is increased in BD patients. We also searched for non-English language studies and were not able to identify any relevant studies based on title and abstract review. We hope this search will serve to limit the non-English language publication bias.

4.3. Limitations of the Study. Our study has some limitations. Unknown factors can always be a source of bias and may cause confounding. Observer bias may have contributed as most of the papers did not mention if the TTE operator was blinded to the case-control status of the patients. Publication bias against negative studies could not be excluded.

In clinical practice, DD diagnosis is based on several TTE values and not one solitary abnormality and the reliance on only one abnormal value may have elevated the degree of presence of DD in our paper. Also, our values do not meet any predefined cut-off values favoring DD but just that the TTE indices are more inclined towards $\mathrm{DD}$ in $\mathrm{BD}$ patients and these are statistically significant. We were unable to report on the grade of DD as it was mostly not reported or not possible to combine in the analysis. As we discussed earlier, the grade of DD is important from a prognostic perspective.

We did note that our cases had slightly higher DBP values than controls. Controls had higher HDL levels and BMI. While BMI was higher in the control group, its average was within the normal range and likely was not a contributing factor to the results. And even if it were, it would likely have only served to reduce the difference between the two groups by increased values of the TTE measurements in the control population. HDL level at present seems to have no robust influence on DD. It may be argued that the higher DBP might have confounded the results but we do not believe so due to the fact that average DBP was within the normal range and that SBP is the more important determinant from the pathology and management perspective, and it was similar in the two groups [59].

As there was a considerable amount of heterogeneity in the papers used in the meta-analysis, we utilized randomeffects models to limit the effects of heterogeneity on the results of our meta-analysis. This heterogeneity was a result of different patient populations, clinical environment, TTE performers, BD severity, and procedure of control selection. The results of this aggregate data meta-analysis may not be applicable to the singular patient as no individual level data was ascertainable. Most of the data is confined to studies from Turkey which is the country that has the most experience with $\mathrm{BD}$ and thus may not be applicable universally. We must, however, consider that $\mathrm{BD}$ is a disease with geographic risk factors and is expected to be seen more in certain areas than the others. We were also not able to analyze the association between the degree of BD activity or severity and the cardiac manifestations.

\section{Conclusions}

This meta-analysis has combined data from the all the published studies on the topic and identified that DD is increased in patients with $\mathrm{BD}$ by the presence of several TTE parameters favoring DD as compared to controls. It has also identified that LA dimension is increased in $\mathrm{BD}$ patients. EF has also been found to be lower in BD patients. Future longitudinal studies could follow the natural history of cardiac function in $\mathrm{BD}$ patients and see the rate of natural progression of DD to overt CHF and compare it to the trend in the general population.

\section{Disclosure}

This paper was part of the M.S. degree project of the first author.

\section{Competing Interests}

The authors have no funding or competing interests to declare.

\section{References}

[1] C. C. Zouboulis, "Epidemiology of Adamantiades-Behcet's disease," Annales de Médecine Interne, vol. 150, no. 6, pp. 488498, 1999.

[2] S. Yurdakul, I. Gunaydin, Y. Tuzun et al., "The prevalence of Behcet's syndrome in a rural area in northern Turkey," Journal of Rheumatology, vol. 15, no. 5, pp. 820-822, 1988.

[3] J. D. O'Duffy, "Vasculitis in Behcet's disease," Rheumatic Disease Clinics of North America, vol. 16, no. 2, pp. 423-431, 1990.

[4] C. A. Bowles, A. M. Nelson, S. C. Hammill, and J. D. O’Duffy, "Cardiac involvement in Behcet's disease," Arthritis \& Rheumatism, vol. 28, no. 3, pp. 345-348, 1985.

[5] S.-H. Wan, M. W. Vogel, and H. H. Chen, "Pre-clinical diastolic dysfunction," Journal of the American College of Cardiology, vol. 63, no. 5, pp. 407-416, 2014.

[6] K. Sharma and D. A. Kass, "Heart failure with preserved ejection fraction: mechanisms, clinical features, and therapies," Circulation Research, vol. 115, no. 1, pp. 79-96, 2014.

[7] M. Jessup, W. T. Abraham, D. E. Casey et al., "2009 Focused update: ACCF/AHA guidelines for the diagnosis and management of heart failure in adults: a report of the American College 
of Cardiology Foundation/American Heart Association Task Force on practice guidelines: developed in collaboration with the International Society for Heart and Lung Transplantation," Circulation, vol. 119, no. 14, pp. 1977-2016, 2009.

[8] M. M. Redfield, S. J. Jacobsen, J. C. Burnett Jr., D. W. Mahoney, K. R. Bailey, and R. J. Rodeheffer, "Burden of systolic and diastolic ventricular dysfunction in the community: appreciating the scope of the heart failure epidemic," The Journal of the American Medical Association, vol. 289, no. 2, pp. 194-202, 2003.

[9] C. M. Halley, P. L. Houghtaling, M. K. Khalil, J. D. Thomas, and W. A. Jaber, "Mortality rate in patients with diastolic dysfunction and normal systolic function," Archives of Internal Medicine, vol. 171, no. 12, pp. 1082-1087, 2011.

[10] G. C. Kane, B. L. Karon, D. W. Mahoney et al., "Progression of left ventricular diastolic dysfunction and risk of heart failure," The Journal of the American Medical Association, vol. 306, no. 8, pp. 856-863, 2011.

[11] N. Achong, S. Wahi, and T. H. Marwick, "Evolution and outcome of diastolic dysfunction," Heart, vol. 95, no. 10, pp. 813818, 2009.

[12] H. Fukuta and W. C. Little, "Diagnosis of diastolic heart failure," Current Cardiology Reports, vol. 9, no. 3, pp. 224-228, 2007.

[13] R. F. Yturralde and W. H. Gaasch, "Diagnostic criteria for diastolic heart failure," Progress in Cardiovascular Diseases, vol. 47, no. 5, pp. 314-319, 2005.

[14] F. Aslam, S. J. Bandeali, N. A. Khan, and M. Alam, "Diastolic dysfunction in rheumatoid arthritis: a meta-analysis and systematic review," Arthritis Care \& Research, vol. 65, no. 4, pp. 534-543, 2013.

[15] S. Yurdakul, V. A. Erdemir, Y. Tayyareci, O. Yildirimturk, M. Salih Gurel, and S. Aytekin, "Subclinical left and right ventricular systolic dysfunction in Behcet's disease: a combined tissue doppler and velocity vector imaging study," Journal of Clinical Ultrasound, vol. 41, no. 6, pp. 347-353, 2013.

[16] E. Aktürk, J. Yağmur, E. Kurtoğlu et al., "Left atrial volume and function in patients with Behcet's disease assessed by real-time three-dimensional echocardiography," European Heart Journal Cardiovascular Imaging, vol. 13, no. 8, pp. 650-655, 2012.

[17] T. Karabag, M. Aydin, S. M. Dogan et al., "Investigation of the atrial electromechanical delay duration in Behcet patients by tissue Doppler echocardiography," European Heart Journal Cardiovascular Imaging, vol. 13, no. 3, pp. 251-256, 2012.

[18] V. Cobankara, A. Guclu, O. Kuru et al., "Evaluation of biventricular myocardial performance index in patients with Behçet's disease," Journal of International Medical Research, vol. 40, no. 1, pp. 328-332, 2012.

[19] J. Yagmur, S. Sener, N. Acikgoz et al., "Subclinical left ventricular dysfunction in Behcet's disease assessed by twodimensional speckle tracking echocardiography," European Journal of Echocardiography, vol. 12, no. 7, pp. 536-541, 2011.

[20] F. Koc, S. Koc, J. Yuksek et al., "Is diastolic dysfunction associated with atrial electrocardiographic parameters in Behçet's disease?" Acta Cardiologica, vol. 66, no. 5, pp. 607-612, 2011.

[21] E. B. Kaya, H. Yorgun, A. Akdogan et al., "Heart-rate recovery index is impaired in Behçet's disease," Texas Heart Institute Journal, vol. 36, no. 4, pp. 282-286, 2009.

[22] Y. Tavil, M. A. Ozturk, N. Sen et al., "The assessment of cardiac functions by tissue Doppler-derived myocardial performance index in patients with Behcet's disease," Clinical Rheumatology, vol. 27, no. 3, pp. 309-314, 2008.
[23] I. Ikonomidis, K. Aznaouridis, A. Protogerou et al., "Arterial wave reflections are associated with left ventricular diastolic dysfunction in Adamantiades-Behçet's disease," Journal of Cardiac Failure, vol. 12, no. 6, pp. 458-463, 2006.

[24] B. Yavuz, L. Sahiner, A. Akdogan et al., "Left and right ventricular function is impaired in Behçet's disease," Echocardiography, vol. 23, no. 9, pp. 723-728, 2006.

[25] N. Baris, T. Okan, O. Gurler et al., "Evaluation of left ventricular diastolic dysfunction with conventional and current Doppler techniques in Behcet's disease," Clinical Rheumatology, vol. 25, no. 6, pp. 873-876, 2006.

[26] A. Bozkurt, O. Akpinar, S. Uzun, A. Akman, D. Arslan, and A. Birand, "Echocardiographic findings in patients with Behçet's disease," American Journal of Cardiology, vol. 97, no. 5, pp. 710$715,2006$.

[27] S. E. Tunc, A. Dogan, O. Gedikli, C. Arslan, and M. Sahin, "Assessment of aortic stiffness and ventricular diastolic functions in patients with Behçet's disease," Rheumatology International, vol. 25, no. 6, pp. 447-451, 2005.

[28] E. Topal, R. Ozdemir, Y. Aksoy et al., “Tissue doppler velocities of the right and left ventricles and their association with Creactive protein and homocysteine levels in Behcet's disease," American Journal of Cardiology, vol. 96, no. 12, pp. 1739-1742, 2005.

[29] I. Ikonomidis, J. Lekakis, K. Stamatelopoulos, N. Markomihelakis, P. G. Kaklamanis, and M. Mavrikakis, "Aortic elastic properties and left ventricular diastolic function in patients with Adamantiades-Behcet's disease," Journal of the American College of Cardiology, vol. 43, no. 6, pp. 1075-1081, 2004.

[30] C. Gürgün, E. Ercan, C. Ceyhan et al., "Cardiovascular involvement in Behçet's disease," Japanese Heart Journal, vol. 43, no. 4, pp. 389-398, 2002.

[31] K. Gemici, I. Baran, S. Gullulu, A. R. Kazazoglu, J. Cordan, and Z. Ozer, "Evaluation of diastolic dysfunction and repolarization dispersion in Behcet's disease," International Journal of Cardiology, vol. 73, no. 2, pp. 143-148, 2000.

[32] O. Kirimli, O. Aslan, O. Göldeli et al., "Heart rate variability, late potentials and QT dispersion as markers of myocardial involvement in patients with Behcet's disease," Canadian Journal of Cardiology, vol. 16, no. 3, pp. 345-351, 2000.

[33] S. Morelli, C. Perrone, L. Ferrante et al., "Cardiac involvement in Behcet's disease," Cardiology, vol. 88, no. 6, pp. 513-517, 1997.

[34] B. Komsuoglu, Ö. Göldeli, K. Kulan et al., "Doppler evaluation of left ventricular diastolic filling in Behçet's disease," International Journal of Cardiology, vol. 47, no. 2, pp. 145-150, 1994.

[35] M. Calguneri, B. Erbas, S. Kes, and Y. Karaaslan, "Alterations in left ventricular function in patients with Behcet's disease using radionuclide ventriculography and Doppler echocardiography," Cardiology, vol. 82, no. 5, pp. 309-316, 1993.

[36] M. Özkan, O. Emel, M. Özdemir et al., "M-mode, 2-D and doppler echocardiographic study in 65 patients with Behçet's syndrome," European Heart Journal, vol. 13, no. 5, pp. 638-641, 1992.

[37] D. F. Stroup, J. A. Berlin, S. C. Morton et al., "Meta-analysis of observational studies in epidemiology: a proposal for reporting," The Journal of the American Medical Association, vol. 283, no. 15, pp. 2008-2012, 2000.

[38] "Criteria for diagnosis of Behcet's disease. International Study Group for Behcet's Disease," The Lancet, vol. 335, no. 8697, pp. 1078-1080, 1990. 
[39] G. A. Wells, B. Shea, D. O'Connell et al., The Newcastle-Ottawa Scale (NOS) for Assessing the Quality of Nonrandomized Studies in Meta-Analyses, Department of Epidemiology and Community Medicine, University of Ottawa, Ottawa, Canada, http://www.ohri.ca/programs/clinical_epidemiology/oxford .htm.

[40] M. Galderisi, "Diastolic dysfunction and diastolic heart failure: diagnostic, prognostic and therapeutic aspects," Cardiovascular Ultrasound, vol. 3, article 9, 2005.

[41] S. Bangalore, S.-S. Yao, and F. A. Chaudhry, "Role of left atrial size in risk stratification and prognosis of patients undergoing stress echocardiography," Journal of the American College of Cardiology, vol. 50, no. 13, pp. 1254-1262, 2007.

[42] R. Yağc1, Y. Totan, E. Özlük, H. Özyurt, G. Akbay, and M. Ekşioğlu, "Serum levels of natriuretic peptides in patients with Behcet's disease," Clinical Rheumatology, vol. 27, no. 9, pp. 11531158, 2008.

[43] E. Seyahi, M. Baskurt, M. Melikoglu et al., "The estimated pulmonary artery pressure can be elevated in Behçet's syndrome," Respiratory Medicine, vol. 105, no. 11, pp. 1739-1747, 2011.

[44] K. Yoshikawa, H. Hori, S. Fukunaga, E. Tayama, and S. Aoyagi, "Aortic root replacement in Behçet disease," Asian Cardiovascular and Thoracic Annals, vol. 15, no. 6, pp. 521-523, 2007.

[45] I. H. Güllü, M. Benekli, H. Müderrisoğlu et al., "Silent myocardial ischemia in Behçet's disease," Journal of Rheumatology, vol. 23, no. 2, pp. 323-327, 1996.

[46] F. Kosar, I. Sahin, H. Gullu, and S. Cehreli, "Acute myocardial infarction with normal coronary arteries in a young man with the Behcet's disease," International Journal of Cardiology, vol. 99, no. 2, pp. 355-357, 2005.

[47] S. Yilmaz, G. Celik, and S. E. Esmen, "Assessment of arterial stiffness in patients with inactive and active Behçet's disease," Scandinavian Journal of Rheumatology, vol. 43, no. 1, pp. 63-69, 2014.

[48] E. Osto, S. Piaserico, A. Maddalozzo et al., "Impaired coronary flow reserve in young patients affected by severe psoriasis," Atherosclerosis, vol. 221, no. 1, pp. 113-117, 2012.

[49] M. Caliskan, H. Gullu, S. Yilmaz et al., "Cardiovascular prognostic value of vascular involvement in Behcet's disease," International Journal of Cardiology, vol. 125, no. 3, pp. 428-430, 2008.

[50] F. Atzeni, P. Sarzi-Puttini, G. De Blasio, L. Delfino, L. Tomasoni, and M. Turiel, "Preclinical impairment of coronary flow reserve in patients with rheumatoid arthritis," Annals of the New York Academy of Sciences, vol. 1108, pp. 392-397, 2007.

[51] R. Montisci, A. Vacca, P. Garau et al., "Detection of early impairment of coronary flow reserve in patients with systemic sclerosis," Annals of the Rheumatic Diseases, vol. 62, no. 9, pp. 890-893, 2003.

[52] D. D. Correa de Sa, D. O. Hodge, J. P. Slusser et al., "Progression of preclinical diastolic dysfunction to the development of symptoms," Heart, vol. 96, no. 7, pp. 528-532, 2010.

[53] T. S. M. Tsang, M. E. Barnes, K. R. Bailey et al., "Left atrial volume: important risk marker of incident atrial fibrillation in 1655 older men and women," Mayo Clinic Proceedings, vol. 76, no. 5, pp. 467-475, 2001.

[54] M. Aktoz, M. Yilmaztepe, E. Tatli, F. N. Turan, E. G. Ümit, and A. Altun, "Assessment of ventricular and left atrial mechanical functions, atrial electromechanical delay and $\mathrm{P}$ wave dispersion in patients with scleroderma," Cardiology Journal, vol. 18, no. 3 , pp. 261-269, 2011.
[55] A. J. J. T. Rein, C. P. O’Donnell, S. D. Colan, and G. R. Marx, "Tissue velocity Doppler assessment of atrial and ventricular electromechanical coupling and atrioventricular time intervals in normal subjects," American Journal of Cardiology, vol. 92, no. 11, pp. 1347-1350, 2003.

[56] S. M. Dogan, M. Aydin, M. Gursurer et al., "The increase in $\mathrm{P}$-wave dispersion is associated with the duration of disease in patients with Behçet's disease," International Journal of Cardiology, vol. 124, no. 3, pp. 407-410, 2008.

[57] V. Palmieri, C. Russo, and J. N. Bella, “Treatment of isolated left ventricular diastolic dysfunction in hypertension: reaching blood pressure target matters," Hypertension, vol. 55, no. 2, pp. 224-225, 2010.

[58] A. Hassanein, A. Desai, A. Verma et al., "EXCEED: exforge ${ }^{\circledR}$ intensive control of hypertension to evaluate efficacy in diastolic dysfunction: Study rationale, design, and participant characteristics," Therapeutic Advances in Cardiovascular Disease, vol. 3, no. 6, pp. 429-439, 2009.

[59] T. E. Strandberg and K. Pitkala, "What is the most important component of blood pressure: systolic, diastolic or pulse pressure?" Current Opinion in Nephrology and Hypertension, vol. 12, no. 3, pp. 293-297, 2003. 


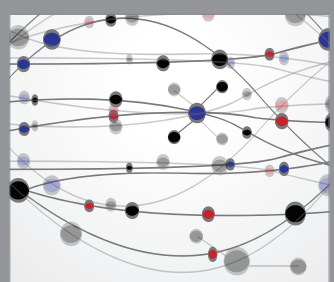

The Scientific World Journal
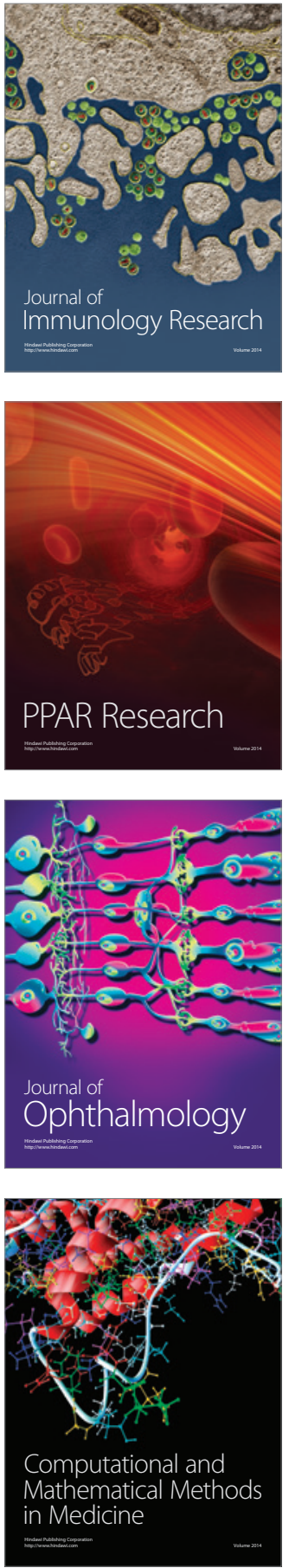

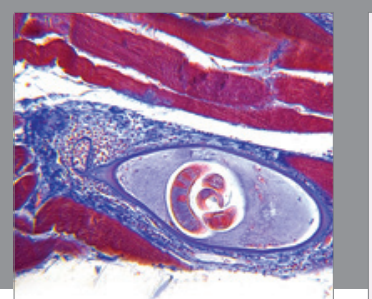

Gastroenterology Research and Practice

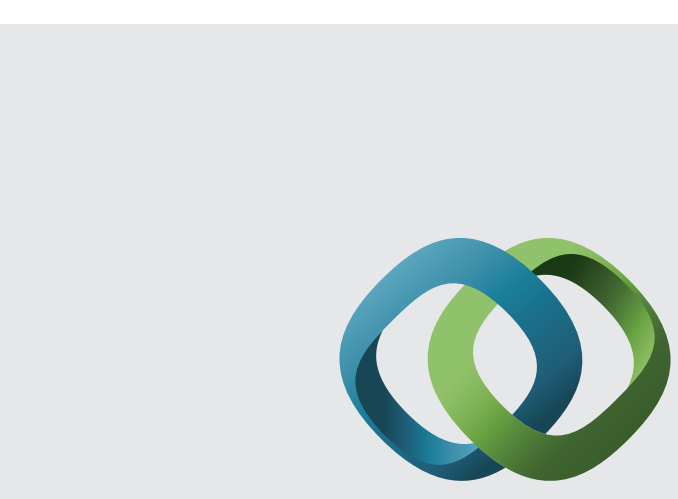

\section{Hindawi}

Submit your manuscripts at

http://www.hindawi.com
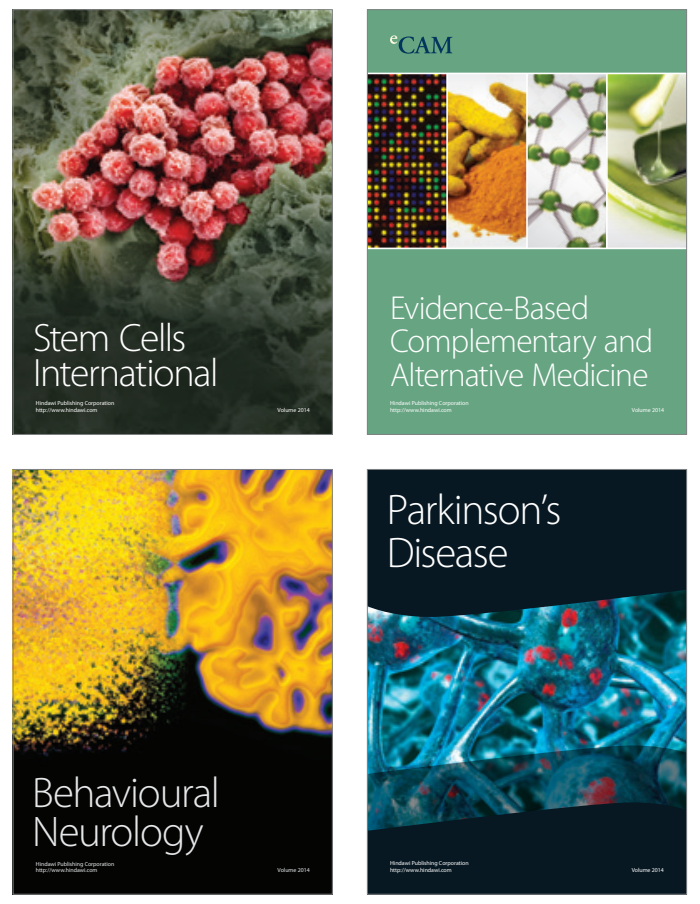
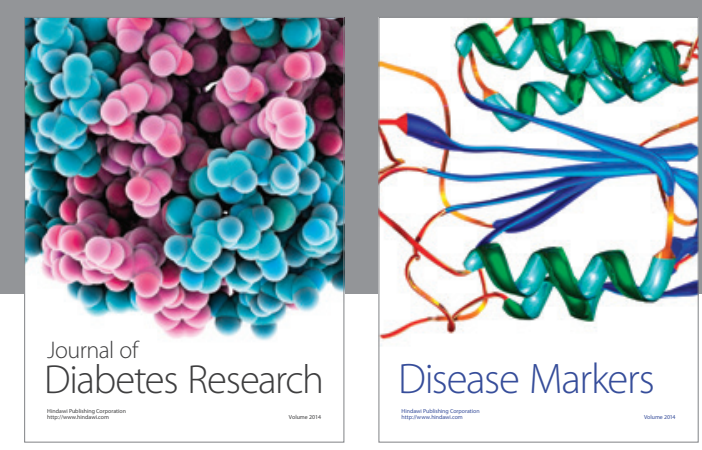

Disease Markers
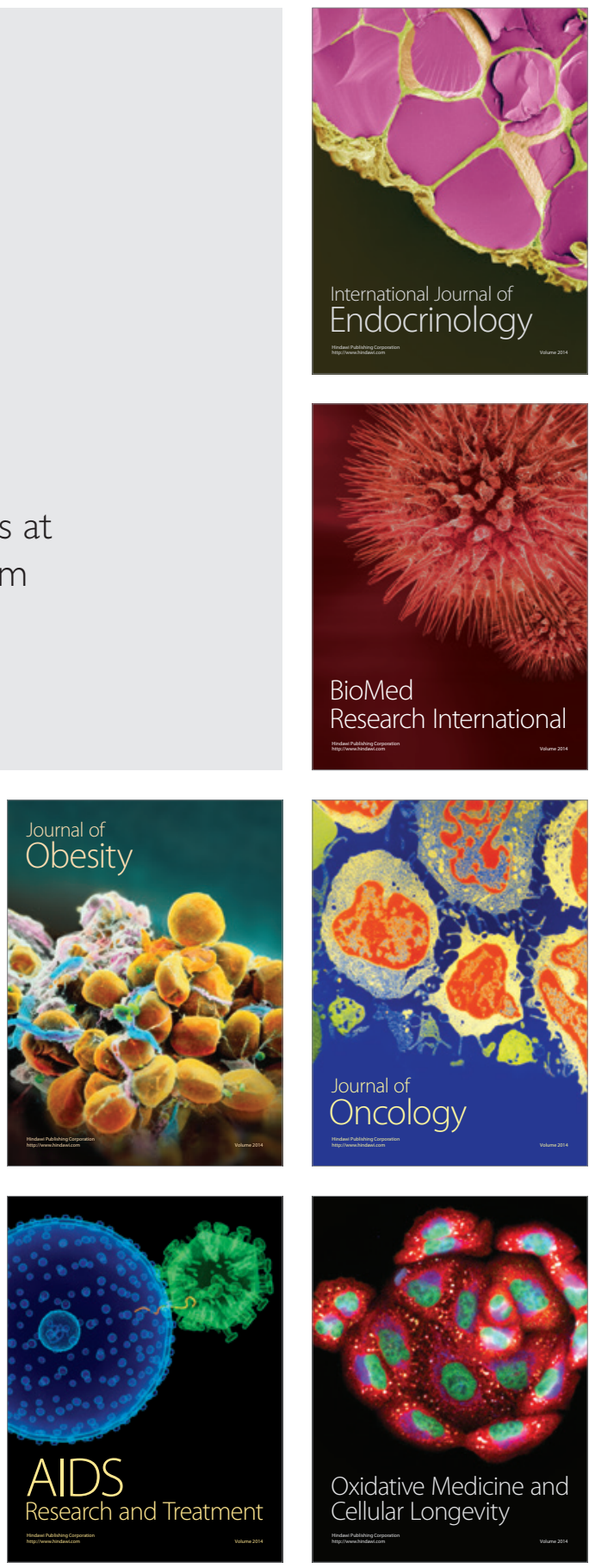